\title{
MS15-02 | High-Pressure Synthesis ANd Properties of Iron Oxides
}

Ovsyannikov, Sergey (Bayerisches Geoinstitut, Universität Bayreuth, Bayreuth, GER); Bykov, Maxim (Bayerisches Geoinstitut, Universität Bayreuth,, Bayreuth, GER); Bykova, Elena (Bayerisches Geoinstitut, Universität Bayreuth, Bayreuth, GER); Glazyrin, Konstantin (Deutsches Elektronen-Synchrotron (DESY), Hamburg, GER); Manna, Rudra (Institute of Physics, University of Augsburg, Augsburg, AUT); Tsirlin, Alexander (Institute of Physics, University of Augsburg, Bayreuth, GER); Cerantola, Valerio (Bayerisches Geoinstitut, Universität Bayreuth, Bayreuth, GER); Kupenko, Ilya (ESRF-The European Synchrotron, Grenoble, FRA); Kurnosov, Alexander (Bayerisches Geoinstitut, Universität Bayreuth, Bayreuth, GER); Kantor, Innokenty (ESRF-The European Synchrotron, Grenoble, GER); Pakhomova, Anna (Deutsches Elektronen-Synchrotron (DESY), Hamburg, GER); Chuvashova, Irina (Bayerisches Geoinstitut, Universität Bayreuth, Bayreuth, GER); Chumakov, Aleksandr (ESRF-The European Synchrotron, Grenoble, GER); Rüffer, Rudolf (ESRF-The European Synchrotron, Grenoble, FRA); McCammon, Catherine (Bayerisches Geoinstitut, Universität Bayreuth, Bayreuth, GER); Dubrovinsky, Leonid (Bayerisches Geoinstitut, Universität Bayreuth, Bayreuth, GER)

Iron oxides are common and fundamentally important materials for natural sciences. In this presentation, we will review a progress on high-pressure high-temperature (HP-HT) synthesis of novel iron oxides, like $\mathrm{Fe}_{4} \mathrm{O}_{5}, \mathrm{Fe}_{5} \mathrm{O}_{6}$, $\mathrm{Fe}_{7} \mathrm{O}_{9}, \mathrm{Fe}_{5} \mathrm{O}_{7}, \mathrm{Fe}_{9} \mathrm{O}_{11}, \mathrm{FeO}_{2}$, and others, and present recent results on high-pressure low-temperature behaviour of one of them, namely, $\mathrm{Fe}_{4} \mathrm{O}_{5}$. We will present the results of combined single-crytal X-ray diffraction, Mossbauer spectroscopy and magnetization measurements, performed on $\mathrm{Fe}_{4} \mathrm{O}_{5}$ samples under high pressure at low temperature. We will show how the applied pressure that tunes the distancies between the neighbouring iron atoms can switch the charge-ordering type between the trimers and dimers. We will show that the chargeordering processes in iron oxides are predetermined not only by spin and sharge interactions, but also by "chemical" interactions between the neighbouring iron atoms. We synthesized large single crystals of $\mathrm{Fe}_{4} \mathrm{O}_{5}$ using multi-anvil high-pressure high-temperature synthesis facilities, and the major part of the studies has been done on single crystals. We will report and discuss the first P-T phase diagram of $\mathrm{Fe}_{4} \mathrm{O}_{5}$. We also discuss some preliminary results for the other iron oxides. 